\title{
A MODELING FRAMEWORK FOR COLLABORATIVE NETWORKED ORGANIZATIONS
}

\author{
Luis M. Camarinha-Matos ${ }^{1}$; Hamideh Afsarmanesh ${ }^{2}$ \\ ${ }^{1}$ New University of Lisbon, PORTUGAL, cam@uninova.pt \\ ${ }^{2}$ University of Amsterdam,THE NETHERLANDS, hamideh@science.uva.nl
}

Collaborative networked organizations are complex entities whose proper understanding, design, implementation, and management require the integration of different modeling perspectives. A comprehensive modeling framework is therefore proposed as a first step towards the elaboration of a reference model for collaborative networks. Modeling tools and theories developed in other disciplines are also analyzed in terms of their potential applicability in this domain.

\section{INTRODUCTION}

Modeling is one of the key activities in understanding, designing, implementing, and operating systems. Modeling is at the very heart of any scientific and engineering activity. When a team of researchers or system designers develop a new system, the output of the design phase is a model or set of models of the system to be implemented. A model, as an abstract representation of the intended system, will then be used to guide the implementation. Due to a number of practical contingencies, the implemented system might show some (minor) differences regarding the original model (usually the case). Furthermore, a model is also very useful in order to supervise (manage) the operation of the developed system during its life cycle. Complementarily, a model can also be used to predict the behavior of the system being developed or managed.

As in any other scientific discipline or engineering branch, collaborative networked organizations (CNOs) require the development of models, not only as a help to better understand the area, but also as the basis for the development of methods and tools for better decision-making. In fact proper decision-making in all phases of the CNO life cycle needs to be based on well argued and verified models and methodologies. These models and methodologies constitute the basis for the ICT-based support for business and organizational development and operation, as well as the base for education, training, and effective operation of CNOs. 
CNOs are complex systems, emerging in many forms in different application domains, and consist of many facets whose proper understanding requires the contribution from multiple disciplines. However, an analysis of past modeling efforts indicates that practitioners and researchers are not fully aware of a comprehensive spectrum of suitable modeling processes, tools, and methodologies. For instance, very often modeling is restricted to a "processes view" (e.g. SCOR type of models for traditional supply chains). Or they stick with one approach such as using UML even though it might not the most appropriate approach for all or a part of the modeling effort.

This situation is however improving and lately some theories and paradigms defined elsewhere have been suggested by several research groups as promising tools to help understand and characterize emerging collaborative organizational forms [1], [2], [3]. Nevertheless, it is unlikely that any of these theories and modeling methods will cover all needs of $\mathrm{CNO}$; they can be used as a starting point but extensions or adaptations are needed. There is no single formal modeling tool/ approach that adequately covers all perspectives - no "universal language" for all problems. And yet existing knowledge on diverse manifestations of "traditional" collaborative networks is quite fragmented, being urgent to proceed with an integration and formalization effort. Nevertheless, purely formal methods in addition to being hard to apply are also difficult to follow by those not familiar with such methods.

Dissemination and communication is one important purpose for modeling CNOs. As such, we must acknowledge that this area is addressed by a large variety of people with different backgrounds; not all of them possess a strong formal background, and even many of the ICT practitioners do not have a formal education on computer engineering or computer science. This might suggest, in some cases, the appropriateness of semi-formal methods. On the other hand, new forms of collaborative networks and new patterns of behavior are being invented and explored, for which it is not feasible to develop fully consistent formal models at start. In these cases, semi-formal models, or even informal analogies as represented by metaphors, can provide valuable insights towards a preliminary level of understanding of new collaborative forms.

This paper analyses a set of relevant modeling needs for CNOs, considers a collection of tools and theories developed elsewhere that might be useful here, and suggests a modeling framework for CNOs integrating multiple perspectives.

\section{MODELING NEEDS IN CNO}

In the context of a complex system like a CNO, modeling is fundamental for understanding, managing, simulating / predicting the behavior of CNOs, and certainly also for software development. For instance, in the VOSTER project [4], several purposes for modeling in this domain were also considered. In ECOLEAD a large number, though incomplete, of modeling purposes were identified for various kinds of CNOs. Based on those examples, Figure 1 illustrates some of the important questions a modeler may pose when attempting to model a virtual organizations breeding environment (VBE). 


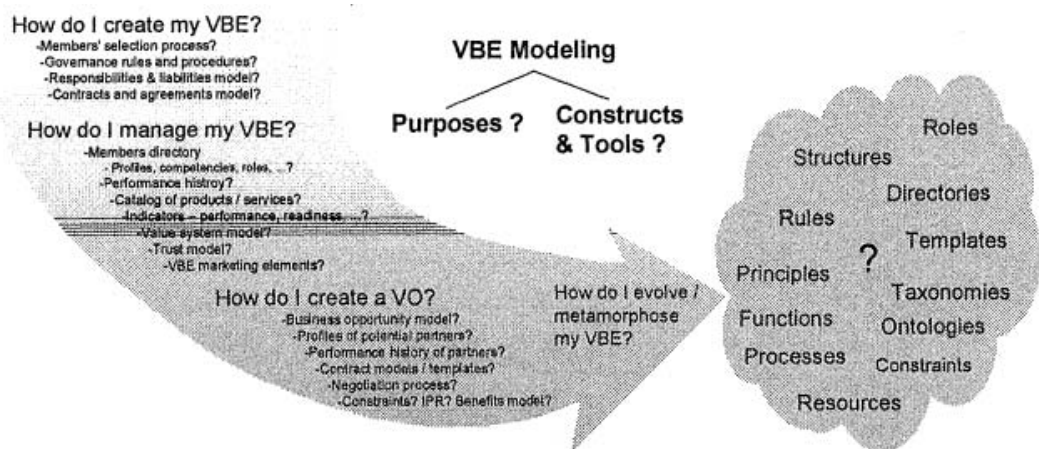

Figure 1 - Examples of modeling purposes in VBE

Certainly many other relevant questions may be asked in relation to a VBE. Similarly, for VO management a large number of modeling purposes are typically considered (Figure 2).

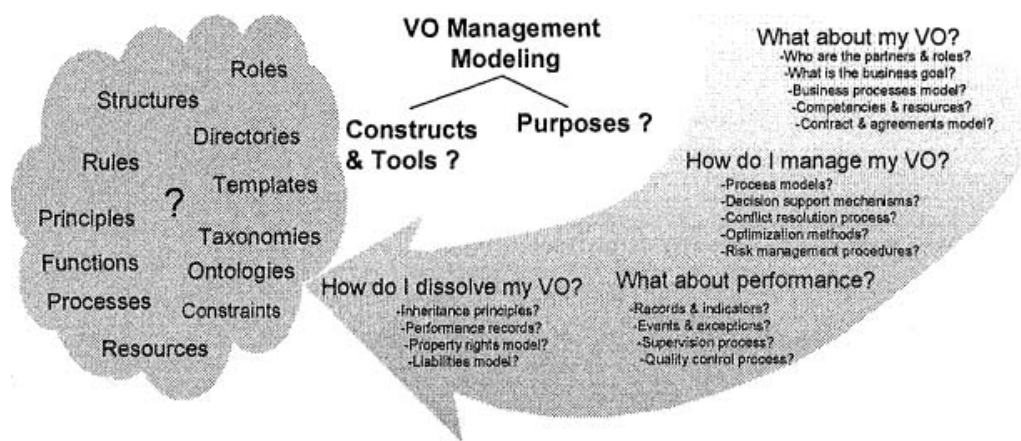

Figure 2 - Examples of modeling purposes in VO management

In the same way many purposes are identifiable for Professional Virtual Communities (PVCs) and other forms of CNOs. Given this large diversity of modeling purposes, which also leads to different types of models, it is important to establish a framework for modeling that structures and guides the modeling process.

\section{CNO MODELING DIMENSIONS}

As a first attempt to reach a comprehensive modeling framework for CNO modeling, four dimensions are proposed as follows:

- Structural dimension. This perspective addresses the structure or composition of the CNO in terms of its constituting elements (participants and their relationships) as well as the roles performed by those elements and other characteristics of the network nodes such as the location, time, etc. This perspective is used in many disciplines (e.g. systems engineering, software engineering, economy, politics, cognitive sciences, manufacturing), although with different "wording" and diversified tools. 
- Componential dimension. This dimension focuses on the individual tangible/intangible elements in the CNO's network, e.g. the resource composition such as human elements, software and hardware resources, information and knowledge. Not all these elements are "physical" in a strict sense but rather represent the "things" of which the network is built of. Furthermore, the componential dimension also consists of ontology and the description of the information/knowledge repositories that pertain to the CNO.

- Functional dimension. This perspective addresses the "base operations" available at the network and the execution of time-sequenced flows of operations (processes and procedures) related to the "operational phase" of the CNO's life cycle.

- Behavioral dimension. This dimension addresses the principles, policies, and governance rules that drive or constrain the behavior of the CNO and its members over time. Included here are elements such as principles of collaboration and rules of conduct, contracts, conflict resolution policies, etc.

These specific dimensions are chosen for the reason of their "near-orthogonality" in the sense that if elements in different dimension are bound to each other, then changes in one dimension affect the elements of the other dimensions, weakly across some region of relevance. For example, extending the number of workers in one organization (a physical element modeling an organization) may change the options in a process workflow (a functional element modeling that organization). As such, every CNO can be comprehensively defined (modeled) by the collection of its four models, as well as a set of bindings across the constituents of those models. Every model represents specific (and orthogonal) aspects/perspective/dimension of a CNO.

Two examples for bindings follow: 1- Dependencies and bounds between the physical components (e.g. the personnel) and the structural model counterpart (e.g. the role and skill of the personnel) within a CNO. 2- Connection between an organization's structural component (e.g. rights/duties of the organization in a VO) and the behavioral model counterpart (e.g. the contract component in the VO).

The suggested dimensions are still very general and it is important to consider a finer level of granularity; in other words, to consider a set of sub-dimensions for each dimension. Therefore the following set of sub-dimensions is initially proposed for a $\mathrm{CNO}$ modeling framework:

\section{Structural dimension}

a) Actors / relationships - identifying all the participating actors (nodes) in the network as well as their inter-relationships (arcs). The actors can be enterprises, other types of organizations, or people. Two (or more) actors can be linked through a number of different types of relationships, e.g. client-supplier, sharing, coauthoring, etc.

b) Roles - describing and characterizing the roles that can be performed by the actors in the network. A role defines an expected behavior for an actor in a given context. Examples of roles include member, coordinator, broker, planner, etc.

2) Componential dimension

a) Hardware / software resources - characterizing the equipment, software, and infrastructures used / shared in the network. In terms of ICT equipment this model can include the architecture of the computer network supporting the collaboration. In 
the case of manufacturing networks it can include the layout of the shared facilities as well as the logistics networks.

b) Human resources - a characterization of the human resources available in the network, namely in terms of their competencies, profile, potential roles they can perform, etc.

c) Information / knowledge resources - under this sub-dimension we can include the repositories of information and knowledge that are shared by the network members or that support the collaboration processes and the networked organization. d) Ontology resources - representing the main (common) ontologies used in the network and that facilitate the mutual understanding among the network members. One example can be the ontology of competencies available in the network.

3) Functional dimension

a) Processes - this sub-dimension is concerned with the processes involved in the main line of activities of the collaboration. Processes represent the main structured part of the operational activities of the network. An example is the distributed business processes in a business oriented CNO.

b) Auxiliary processes - including those processes that are designed to assist the $\mathrm{CNO}$ in terms of its maintenance and improvement of operations. Examples include performance monitoring processes, competencies management processes, etc.

c) Methodologies - typically less formalized than processes, represent the body of practices, procedures, and rules used mainly by human actors in a CNO. They are frequently represented as a semi-structure set of steps (informal enumeration of activities) combined with some structured representation of input / output information. An example can be the methodology to be followed by a broker to announce a business opportunity to the CNO members.

\section{4) Behavioral dimension}

a) Prescriptive behavior - capturing the elements that lie down or prescribe normative guidelines or rules for the proper behavior of the CNO such as (general) principles, strategies, and protocols. An example is a recommendation for CNO members to give preference to network peers when searching for partners for a business opportunity. Another example could be the recommended protocol when negotiating a contract.

b) Obligatory behavior - describing those rules and principles that are mandatory to be followed inside the network. This includes policies, governance values and associated rules, and enforcement steps. An example can be the internal rules used for distribution of benefits or for sharing the operational costs of the network.

c) Constraints and conditions - representing those "environmental features" that limit the context of operation of the CNO and its members. An example is a set of restrictions on the use of intellectual property of one member by other members of the network.

d) Contracts and cooperation agreements - covering both the contracts between the $\mathrm{CNO}$ and external customers and the internal contracts and cooperation agreements among the network members. These models may include both representations understandable to humans and to software systems.

Nevertheless it shall be noted that these sub-dimensions are not exhaustive. They are shown mainly to better characterize, by illustration, the scope of each dimension. 


\section{MAP OF POTENTIAL APPROACHES}

A large number of theories and tools, developed elsewhere, are potentially useful in modeling CNOs or in giving insights to better understand these networks. It would be a matter of practical convenience for the CNO community to have a kind of map or "shopping list" relating such tools and theories to the CNO modeling needs or modeling dimensions. In this direction, Table 1 illustrates the potential applicability of the various theories with respect to the four modeling dimensions introduced in chapter 3. In this table the letters [SD], [CD], [FD], [BD] stand for Structural, Componential, Functional, and Behavioral dimension, respectively.

Table 1 - Some theories and their potential applicability in CNOs

\begin{tabular}{|c|c|}
\hline Theory / Tool & Potential contribution to CNO modeling \\
\hline Benchmarking & $\begin{array}{l}\text { [FD] Assessment of performance in comparison with a reference (benchmark), } \\
\text { including assessment of processes, trustworthiness, and suggestion of best } \\
\text { practices. }\end{array}$ \\
\hline Complexity theories & $\begin{array}{l}\text { [FD] Methods for forecasting emergent behavior, trustworthiness, etc. } \\
\text { [BD] Modeling of emergent behavior in advanced networks. } \\
\text { Qualitative (macro) understanding of CNO's life cycle. }\end{array}$ \\
\hline Decision support & [FD] Give a basis for developing methods to assist humans in decision making. \\
\hline Deontic logic & $\begin{array}{l}\text { [BD] Represent in a formal way aspects such as "it is obligatory that ...", "it is } \\
\text { forbidden that ...", "it is permitted that ...", which can be useful in the } \\
\text { governance of behavior. }\end{array}$ \\
\hline $\begin{array}{l}\text { Distributed group } \\
\text { dynamics }\end{array}$ & $\begin{array}{l}\text { [SD] Focus on inter-group relationships such as power, leadership, etc, } \\
\text { [BD] Analysis of leadership behavior, hostility, compliancy, etc. }\end{array}$ \\
\hline $\begin{array}{l}\text { Diversity in work } \\
\text { teams }\end{array}$ & $\begin{array}{l}\text { [SD] Characterization of the diversity of individuals and cultures found in } \\
\text { CNOs and analysis of the potential induced by this diversity. }\end{array}$ \\
\hline Evolving ontologies & $\begin{array}{l}\text { [CD] To capture the evolution of mutual understanding among members of the } \\
\text { network, but still is offering limited results. }\end{array}$ \\
\hline Federated systems & $\begin{array}{l}\text { [SD] Providing a vision of the CNO as a federation of autonomous, } \\
\text { heterogeneous, and distributed sources of resources (data / information, } \\
\text { services). Relate roles with authorized access to and visibility of resources. } \\
\text { [CD] Distributed data / information repositories. }\end{array}$ \\
\hline $\begin{array}{l}\text { Formal engineering } \\
\text { methods }\end{array}$ & $\begin{array}{l}\text { [SD] [CD] [FD] [BD] Rigorous specifications (mathematical-based) with } \\
\text { potential application in verification and synthesis of systems. Very hard to } \\
\text { apply. }\end{array}$ \\
\hline Formal theories & $\begin{array}{l}\text { [SD] [CD] [FD] [BD] Solve design problems (architecture, protocols, } \\
\text { verification of specifications according to correctness and completeness), } \\
\text { but very hard to develop. If developed for specific perspectives / } \\
\text { subsystems, can contribute to reduce ambiguities and provide a sound basis } \\
\text { for further developments. }\end{array}$ \\
\hline Game theory & $\begin{array}{l}\text { [FD] Can provide concepts for decision-making, e.g.: } \\
-\quad \text { Cooperative game theory: distribution of responsibility and resources. } \\
\text { - Non-cooperative game theory: selection of partners, sustaining } \\
\text { cooperation and trust building. } \\
\text { [BD] Model interactions with formalized incentive structures. }\end{array}$ \\
\hline Graph theory & $\begin{array}{l}\text { [SD] Representation of the structure of the network - topology, routing, activity, } \\
\text { flow. } \\
\text { [FD] Methods to perform computations on flows and optimization. }\end{array}$ \\
\hline $\begin{array}{l}\text { Knowledge } \\
\text { mapping }\end{array}$ & $\begin{array}{l}\text { [CD] Providing visual representations of knowledge which can facilitate } \\
\text { analysis of the CNO and its resources. }\end{array}$ \\
\hline Memetics & $\begin{array}{l}\text { [BD] Help understanding some aspects of the dynamics of evolutionary } \\
\text { processes (cognitive and business) in multi-cultural contexts. }\end{array}$ \\
\hline
\end{tabular}




\begin{tabular}{|c|c|}
\hline Metaphors & $\begin{array}{l}{[\mathrm{SD}][\mathrm{CD}][\mathrm{FD}][\mathrm{BD}] \text { Quick description for human communication namely a }} \\
\text { possible help in expressing complex ill-defined concepts. } \\
\text { Can be used in early stages (conceptual design) as long as they are not taken too } \\
\text { literally. }\end{array}$ \\
\hline $\begin{array}{l}\text { ML/Bayesian } \\
\text { networks }\end{array}$ & $\begin{array}{l}\text { [FD] Use of probabilistic inference to update and revise belief values. } \\
\text { Can support complex inference modeling including rational decision making } \\
\text { systems, value of information and sensitivity analysis. } \\
\text { Causality analysis and support a form of automated learning (parametric } \\
\text { discovery, network discovery, and causal relationships discovery). }\end{array}$ \\
\hline Multi-agent systems & $\begin{array}{l}\text { [FD] [BD] Model societies of autonomous, distributed and heterogeneous } \\
\text { entities, giving insights on how these societies can be organized and their } \\
\text { behavior regulated through norms and institutions. } \\
\text { [FD] Brokering, coalition formation and negotiation. } \\
\text { [BD] Simulation of self-organizing behavior. }\end{array}$ \\
\hline $\begin{array}{l}\text { Multi-agent } \\
\text { dependency theory }\end{array}$ & $\begin{array}{l}\text { [FD] [SD] Representation of social interactions among agents - dependency } \\
\text { relations, power relations. }\end{array}$ \\
\hline Network analysis & $\begin{array}{l}\text { [SD] [FD] Specialized graph theory-based algorithms for application in } \\
\text { network management systems (mostly applied in telecommunication } \\
\text { networks). }\end{array}$ \\
\hline Portfolio theory & $\begin{array}{l}\text { [FD] Decision making such as in VO creation (to select the optimal VO from a } \\
\text { VBE) }\end{array}$ \\
\hline Real options theory & $\begin{array}{l}\text { [FD] Decision making, e.g. decision to create a VO for a business opportunity, } \\
\text { evaluation of the minimum profitable bid in a call for tenders, etc. }\end{array}$ \\
\hline Scopos theory & $\begin{array}{l}\text { [FD] Understand transformation of information or knowledge from one cultural } \\
\text { and language environment to others in such a way that the understanding } \\
\text { and conception of the source information or knowledge would be the same } \\
\text { for all. }\end{array}$ \\
\hline $\begin{array}{l}\text { Self-organizing } \\
\text { systems }\end{array}$ & $\begin{array}{l}\text { [BD] Understanding and simulation of self-organizing behavior. } \\
\text { [FD] Help in predicting evolution. }\end{array}$ \\
\hline Semiotics & $\begin{array}{l}\text { [BD] Model responsibility relationships and commitments. } \\
\text { Prescribe norms and roles - epistemic, deontic and axiologic. }\end{array}$ \\
\hline $\begin{array}{l}\text { Social network } \\
\text { analysis }\end{array}$ & $\begin{array}{l}\text { [SD] Analysis of social and organizational structure of CNOs, including } \\
\text { provision of a number of metrics. } \\
\text { Ongoing research may lead to useful results on the inclusion of soft-modeling } \\
\text { aspects. }\end{array}$ \\
\hline Soft computing & $\begin{array}{l}\text { [FD] [BD] Represent and exploit the tolerance for imprecision, uncertainty, } \\
\text { partial truth, and approximation. Particularly important to model human } \\
\text { and social aspects. }\end{array}$ \\
\hline Synergetics & [BD] Help understanding emerging behavior and emerging values. \\
\hline $\begin{array}{l}\text { Temporal and } \\
\text { modal logic }\end{array}$ & $\begin{array}{l}\text { [FD] [BD] Focus on the representation of temporal information within a logical } \\
\text { framework. Can be used to model temporal aspects of processes and some } \\
\text { aspects of behavior. }\end{array}$ \\
\hline $\begin{array}{l}\text { Transactions cost } \\
\text { theory }\end{array}$ & [FD] Understand and analyze governance structures based on transaction costs. \\
\hline $\begin{array}{l}\text { Trust building } \\
\text { models }\end{array}$ & $\begin{array}{l}\text { [FD] Organize and systematize the trust building and trust management } \\
\text { processes. }\end{array}$ \\
\hline Web \& text mining & $\begin{array}{l}\text { [FD] Analysis and knowledge discovery from unstructured data: documents in } \\
\text { free text form, web documents. Potential applications include evolution of } \\
\text { ontologies, finding business opportunities, etc. }\end{array}$ \\
\hline
\end{tabular}

In addition to these theories and associated tools, there are other modeling tools that have a generic applicability or have been already widely used in the CNO's community and therefore were not studied in this work. Nevertheless they shall be considered as important candidates to some or all of the modeling dimensions. These generic tools include: 
Table 2 - Additional tools and their potential applicability in CNOs

\begin{tabular}{|l|l|}
\hline Ontology & $\begin{array}{c}{[\mathbf{S D}][\mathbf{C D}][\mathbf{F D}][\mathbf{B D}] \text { Representation of the main CNO concepts and their }} \\
\text { relationships. }\end{array}$ \\
\hline Petri nets & {$[\mathrm{FD}]$ Modeling or processes and auxiliary processes. } \\
\hline Workflow & {$\left[\begin{array}{r}\text { FD] Modeling or processes and auxiliary processes. } \\
\text { [SD] [CD] [FD] [BD] Generic object-oriented modeling tool (graphical language) } \\
\text { with potential application to all dimensions of CNO. However, being a } \\
\text { generic tool, it does not properly capture all specificities of each dimension. }\end{array}\right.$} \\
\hline
\end{tabular}

Figure 3 represents a simplified attempt to establish a map relating theories / tools to the modeling dimensions. This map is not exhaustive and certainly not fully accurate, but just a contribution to give a rough idea of the many possibilities that can be considered.

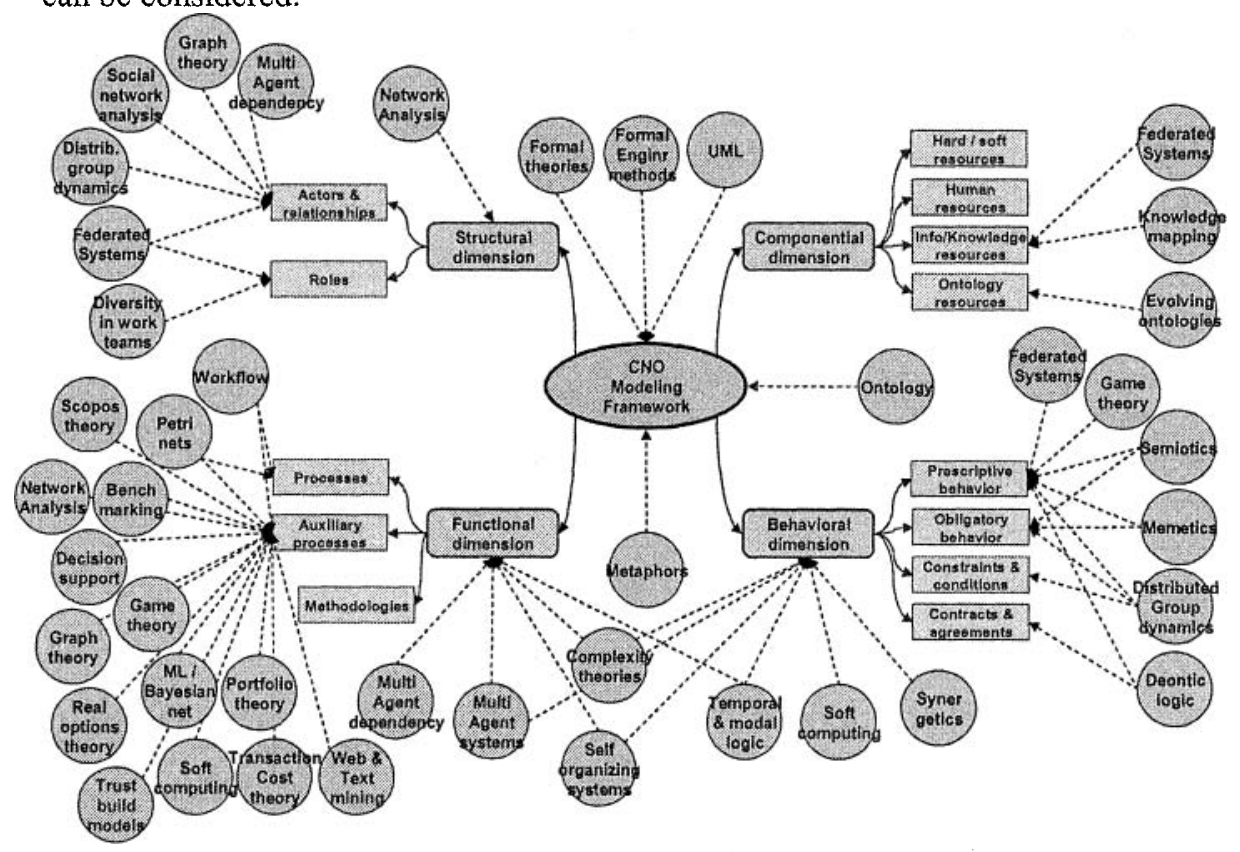

Figure 3 - An attempt to map modeling theories applicable to CNOs

Some theories and tools have a generic nature, others are very specific. For instance, UML or formal engineering methods are quite general and thus potentially applicable to all modeling dimensions; on the other hand, deontic logic is quite specific and potentially useful in the modeling of some aspects of behavior.

As also shown in Figure 3, there are some sub-dimensions for which there is no specialized theory that is particularly suited (e.g. hard/soft resources). Nevertheless there are some generic theories / tools (associated to CNO modeling framework in the center) that are "good for everything", like UML, ontology, etc. Another aspect to consider is that some theories might cover, in part, more than one dimension or sub-dimension. For instance, complexity theories can be linked to the functional and behavioral dimensions. Not all these possibilities are represented in Figure 3.

The suitability of a theory / tool to be applied to a particular modeling perspective also depends on the experience of the modeler with that theory / tool. There are in fact several "gray areas" of applicability. For instance, self-organizing 
systems could, in a limited way, also relate to the structure of the network. Therefore, and in order to not make the map too complex, only what currently seems to be the most important and obvious links are represented.

\section{TOWARDS A HOLISTIC MODELING FRAMEWORK}

When modeling a $\mathrm{CNO}$, it is important to consider both its internal and external aspects (Fig. 4) i.e. how to see the network from inside and from outside.

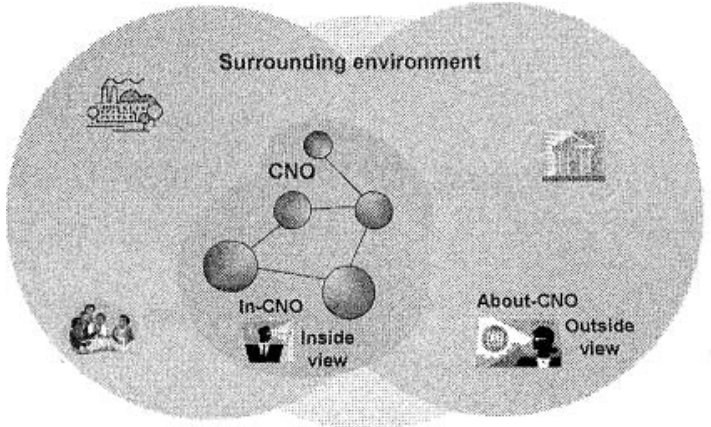

Figure 4 - Two modeling perspectives

We can therefore consider two modeling perspectives or sub-spaces: In-CNO and About-CNO:

In-CNO sub-space. This perspective aims at providing an abstract representation of the CNO from inside, namely the identification of a set of characteristic properties that can together capture the elements constituting CNOs. As discussed in chapter 3, building In-CNO abstract representation is challenging due to the large number of distinct and varied entities, concepts, functionality, rules and regulations, etc. inside the CNOs. In addition to a variety of tangible elements and resources inside the $\mathrm{CNO}$, there are always networks of organizations, in which each node plays a specific role and has heterogeneous relationships with other nodes. Furthermore, there are certain rules of behavior that either constitute the norms, or shall be obeyed by the CNO participants, and needless to say that in every CNO there are a set of activities and functionalities that also need to be abstracted. The four dimensions introduced previously are adequate to model the CNO from the inside perspective.

About-CNO sub-space. This perspective aims at reaching an abstract representation of the CNO as seen from the outside, i.e. which characteristic properties the CNO reveals in its interaction with its "logical" surrounding environment. A CNO as a whole might interact with, influence, and be influenced by a number of "interlocutors", e.g. customers, competitors, external institutions, potential new partners. The interactions between the $\mathrm{CNO}$ and these external entities are quite different, the same as the way each of these entity groups looks at the CNO.

In order to better characterize these differences, the following additional modeling dimensions are proposed for the external or About-CNO perspective:

- Market dimension. This dimension covers both the issues related to the interactions with "customers" (or potential beneficiaries) and "competitors". The customers' facet involves elements such as the transactions and established 
commitments (contracts), marketing and branding, etc. On the competitors' side issues such as market positioning, market strategy, policies, etc. can be considered. The purpose / mission of the $\mathrm{CNO}$, its value proposition, joint identity, etc. are also part of this dimension.

- Support dimension. Under this dimension the issues related to support services provided by third party institutions are to be considered. Examples include certification services, insurance services, training, external coaching, etc.

- Societal dimension. This dimension captures the issues related to the interactions between the $\mathrm{CNO}$ and the society in general. Although this perspective can have a very broad scope, the idea is to model the impacts the CNO has or potentially can have on the society (e.g. impact on employment, economic sustainability of a given region, potential for attraction of new investments) as well as the constraints and facilitating elements (e.g. legal issues, public body decisions, education level) the society provides to the CNO development.

- Constituency dimension. This perspective focuses on the interaction with the universe of potential new members of the CNO, i.e. the interactions with those organizations that are not part of the CNO but that the CNO might be interested in attracting. Therefore, general issues like sustainability of the network, attraction factors, what builds / provides a sense of community, or specific aspects such as rules of adhesion and specific "marketing" policies for members, are considered here.

In addition to these perspectives, a CNO model can be defined at multiple levels of abstraction (model intent perspective). Currently three levels are being considered in our framework:

- General concepts level - that includes the most general concepts and related relationships, common to all CNOs independently of the application domain.

- Specific modeling level - an intermediate level that includes more detailed models focused on different classes of CNOs.

- Implementation modeling level - that represents models of concrete CNOs.

CNO-Life-Cycle perspective. In a typical (long-term) organization, usually its operation stage constitutes its entire livelihood. In other words most successful organizations spend only a negligible fraction of their life time in their setting up and dissolution. Therefore, earlier research on reference modeling for enterprises did not need to elaborate much on the life cycle perspective. But unlike typical organizations, for a wide variety of classes of CNOs their creation stage (as well as their dissolution or metamorphosis) is complex and takes up considerable effort. This is certainly not a negligible fraction of time, and due to the involved complexity, it requires receiving proper attention during the build up of the reference model. Our earlier study of the life cycle stages for CNOs has revealed 4 main stages for the CNO life cycle - Creation, Operation, Evolution, and Metamorphosis / Dissolution.

The ongoing plan for this work is to define "A Reference model for COllaborative Networks" (ARCON). The elaboration of a comprehensive modeling framework, integrating all the above perspectives, is the first step of this initiative.

Figures 5 and 6 combine the life-cycle perspective with the In-CNO and About- 
CNO perspectives respectively.

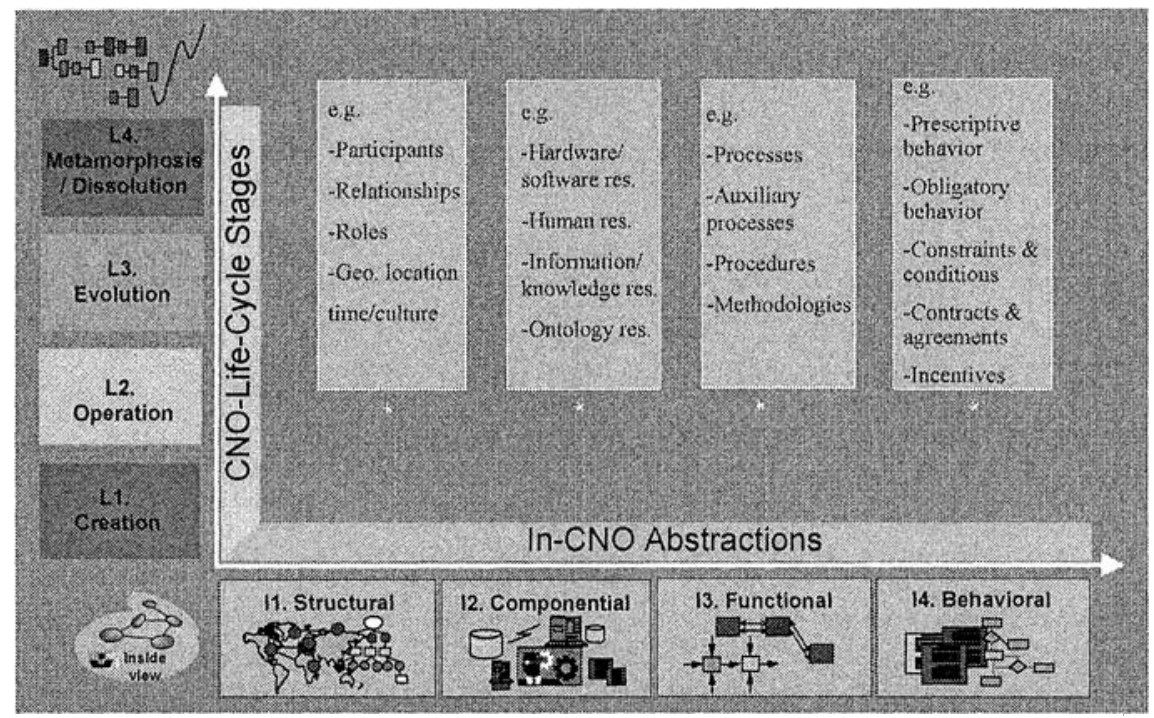

Figure 5-Crossing CNO life cycle and In-CNO abstractions

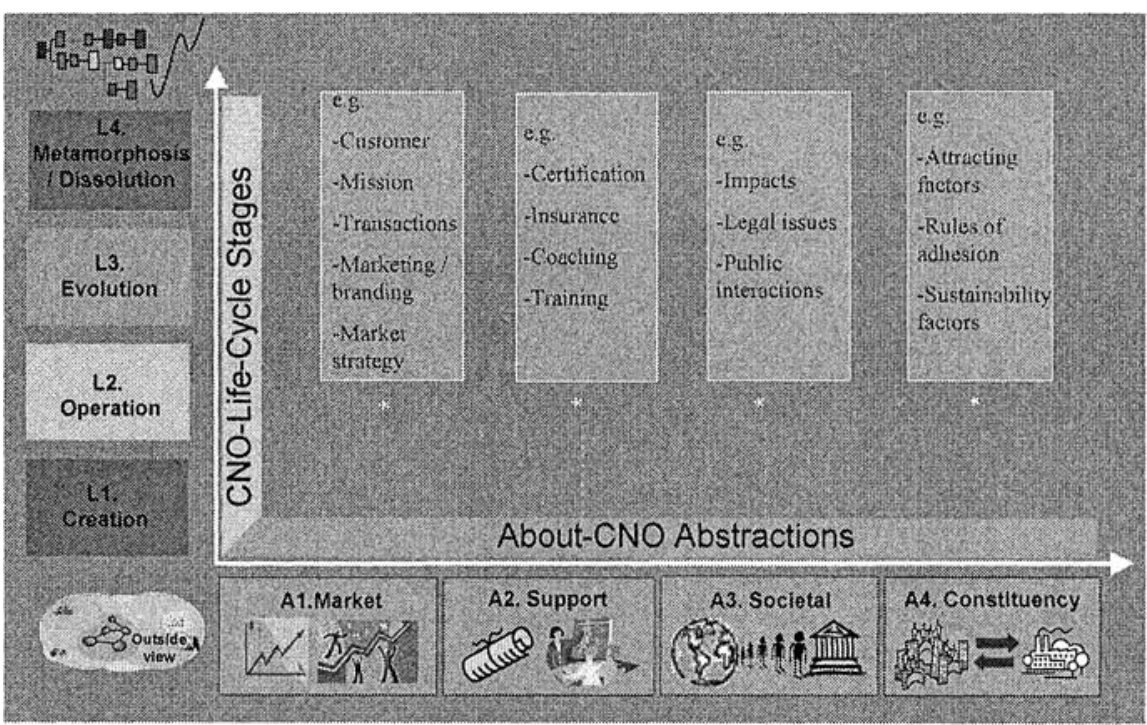

Figure 6 - Crossing CNO life cycle and About-CNO abstractions

Fig. 7 combines the addressed perspectives into a single diagram that summarizes the various perspectives considered in the proposed modeling framework.

\section{CONCLUSIONS}

The establishment of a comprehensive modeling framework for CNOs is a very important basis for the elaboration of a reference model, a base element in the 
consolidation of existing knowledge in this area, and a basis for its consistent further progress. As a contribution in this direction, a modeling framework for CNOs considering multiple perspectives was proposed. Nevertheless it is clear that the establishment of a reference model, able to capture the variety of CNOs and their complexity, is a long term endeavor that needs to start with a careful analysis of the current baseline and definition of related reference modeling frameworks. Current work is focused on the identification of the general modeling elements according to the proposed framework. This analysis is based on different classes of CNOs, namely VO breeding environments, virtual organizations, professional virtual communities, and virtual learning communities.

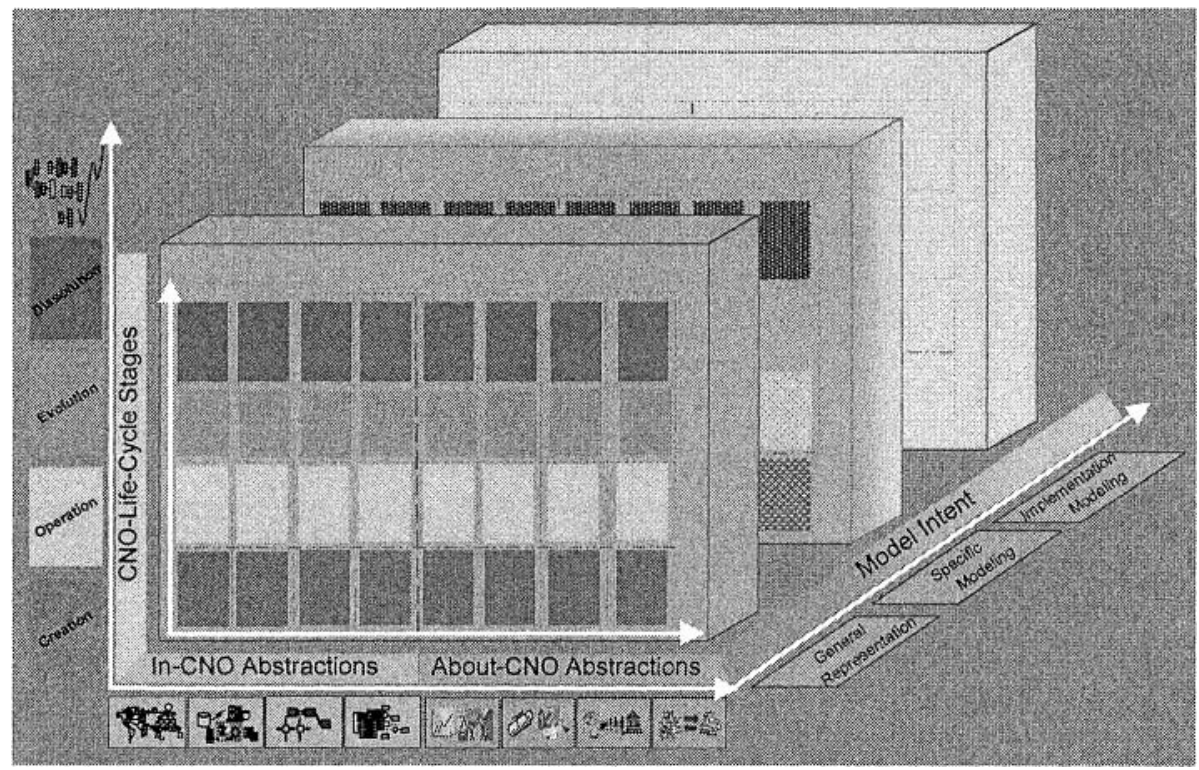

Figure 7 - A modeling framework for CNOs

Acknowledgements. This work was funded in part by the European Commission through the ECOLEAD project. The authors thank the contributions of their partners in this project.

\section{REFERENCES}

1. Camarinha-Matos, L. M; Abreu, A. - Towards a foundation for virtual organizations, in Proceedings of Business Excellence 2003 -Int. Conf. Performance measures, Benchmarking, and Best Practices in New Economy, Guimarães, Portugal, 10-13 Jun 2003.

2. Camarinha-Matos, L. M.; Afsarmanesh, H. - Formal modeling methods for collaborative networks, in Collaborative Networked Organizations $-A$ research agenda for emerging business models, cap. 6.3, Springer, 2004.

3. Eschenbaecher, J; Ellmann, S. - Foundation for networking: A theoretical view on the virtual organization, in Processes and Foundations for Virtual Organizations, Kluwer, 2003.

4. Löh, H.; Zhang, C.; Katzy, B. - Modeling for virtual organizations, in Virtual Organizations - Systems and Practices, Springer, 2005. 